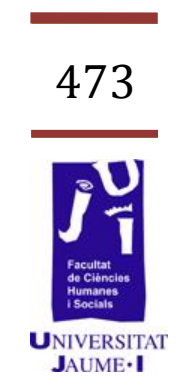

\title{
El acceso a la información periodística en Internet: una propuesta de mejora de las audiotecas de la radio en España
}

Fátima Ramos del Cano fatimaramosdelcano@gmail.com 
I. Resum

La incorporación del medio radio a Internet ha procurado nuevos servicios específicos del entorno digital a sus usuarios, siendo uno de los más valiosos la audioteca. El objetivo de este artículo es conocer el actual sistema de recuperación, almacenamiento y distribución de contenidos radiofónicos de las emisoras COPE, Cadena SER, Onda Cero y Radio Nacional de España e incorporar una propuesta de modelo en base a sus aptitudes y carencias. La metodología puesta en práctica en este trabajo es el análisis de contenido de las audiotecas de las cuatro emisoras principales del sector radiofónico español. Los resultados sugieren una necesaria actualización de este recurso, que puede ser clave en el proceso de reinvención del medio radiofónico que lleva consigo la convergencia digital.

Paraules clau: radio digital, periodismo radiofónico, audioteca, contenidos digitales.

\section{Introducció}

Los archivos sonoros constituyen una importante fuente de información, atesorando su patrimonio un doble valor, el intangible por su contenido y el tangible por su carácter documental y tecnológico. Por eso, "la conservación de los soportes y la preservación de sus contenidos es una tarea integral que implica el rescate y la salvaguarda de la memoria sonora de una comunidad, un pueblo, o una nación». (Rodríguez Resendíz, 2010: 62). "Las grabaciones producidas por la emisora de radio, custodiadas y tratadas técnicamente por el centro de documentación, constituyen un acervo documental sonoro que es una rica muestra de la evolución de la sociedad española en sus diferentes aspectos: social, cultural, político, etc., y forma un archivo histórico de especiales características por lo variado y genuino de su colección integrada por documentos únicos, muy sensibles al paso del tiempo, pero muy valiosos por la espontaneidad e inmediatez de sus contenidos» (Ariza, 2004, p. 30).

Por ello, la gestión de la documentación sonora por parte de las emisoras debería haber estado unida de manera inseparable a la historia y desarrollo de la de la propia radio, una circunstancia que, por desgracia, no ha sido siempre así.

A día de hoy, tal y como podremos constatar más adelante, usuarios y profesionales del medio son conscientes del valor documental/testimonial de la realidad (Cebrián Herreros, 1995: 282) que aporta la información sonora, del patrimonio sonoro de nuestra memoria que éstas albergan, y por eso las audiotecas radiofónicas están nutridas de la producción y transmisión diaria del medio, encontrando entre sus registros además, series radiofónicas, programas, boletines o servicios 
informativos, música, cortes, cuñas, cortinillas o identificaciones de señal. No podemos perder de vista, sin embargo, el salto cualitativo que ha supuesto en este proceso la llegada de Internet, donde la radio se transforma y expande sus espacios (Cebrián, 2008). El acceso a Internet es cada vez mayor y, en consecuencia, también la práctica de escuchar audios a través de la red, tanto vía streaming, como a través de los archivos que las emisoras ponen a disposición de la audiencia para que hagan uso de ella libremente. "Los avances en la compresión de los archivos de audio, la expansión de la banda ancha y el incremento espectacular de periféricos adaptados a la reproducción y almacenamiento de archivos MP3 han facilitado este fenómeno» (Ganzabal Learreta, 2009). Esto es, a la ya labor de recuperación y conservación, se suma ahora la de permitir brindar acceso a sus archivos sonoros no sólo a nivel interno, sino a los propios usuarios.

Esta posibilidad de escucha o radio a la carta que Internet ha posibilitado y que no tienen otras empresas que pueden entrar a competir en la creación de contenidos de audio, debe ser precisamente, el punto de partida para lograr dotar a su audioteca, del deseado valor añadido del que hasta ahora prácticamente carece.

\section{Objectius}

De acuerdo a lo señalado anteriormente, la finalidad de este trabajo se dirige a conocer el estado y funcionamiento de las audiotecas de las principales emisoras españolas, así como el planteamiento que éstas hacen de su presencia en la Red desde el punto de vista de la documentación, esto es, en qué manera pueden ser consideradas fuentes potentes de información retrospectiva, para, posteriormente, ofrecer una propuesta de modelo ejemplar a seguir para un mayor aprovechamiento y gestión de los mismos.

\section{Material i mètode}

El análisis de contenido, como método de investigación para la descripción objetiva, sistemática y cuantitativa del contenido de la comunicación (Berelson, 1952, p. 18), es el elegido en este caso para aproximarnos al estado actual de las audiotecas de las cuatro emisoras de mayor difusión y audiencia de acuerdo con los datos generales del EGM para el último año móvil ${ }^{1}$ (abril 2011- marzo 2012), esto es, Cadena SER, Onda Cero, COPE y RNE.

Por otro lado, para el establecimiento de la relación de valores y elementos de análisis, se ha tomado como referencia el estudio sobre indicadores y buenas prácticas publicado por Javier Guallar y Ernest Abadal (2009) al respecto de las hemerotecas.

${ }^{1}$ Véase: $\underline{\text { http://www.aimc.es/-Datos-EGM-Resumen-General-.html }}$ 
Agrupados en torno a tres grandes apartados como a) Aspectos generales, b) Sistema de consulta y c) Presentación de los resultados, se proponen quince indicadores (Figura 1) que nos permitirán evaluar los servicios que ofrecen, "ya que su presencia, ausencia y grado de desarrollo se pueden considerar valores objetivos en la evaluación de los sistemas» (Guallar, J. y Abadal, E., 2009, p.258).

Tabla 1. Elementos de Valoración. Fuente Guallar, J. y Abadal, $\mathrm{E}^{2}$

\begin{tabular}{|c|c|}
\hline INDICADORES & \\
\hline ASPECTOS GENERALES & $\begin{array}{c}\text { Denominación } \\
\text { Ubicación del acceso } \\
\text { Cobertura temporal } \\
\text { Otros servicios que comprende }\end{array}$ \\
\hline $\begin{array}{l}\text { SISTEMA DE } \\
\text { CONSULTA }\end{array}$ & $\begin{array}{c}\text { Operadores de consulta } \\
\text { Frase literal } \\
\text { Acotación temporal } \\
\text { Acotación en colecciones } \\
\text { Acotación en campos } \\
\text { determinados } \\
\text { Acotación en formatos }\end{array}$ \\
\hline $\begin{array}{l}\text { PRESENTACIÓN DE LOS } \\
\text { RESULTADOS }\end{array}$ & $\begin{array}{l}\text { Gestión de listas de resultados } \\
\text { Agrupación por categorías } \\
\text { Identificación de los términos de } \\
\text { búsqueda } \\
\text { Presentación de documentos } \\
\text { relacionados } \\
\text { Gestión de los resultados }\end{array}$ \\
\hline
\end{tabular}

\section{Resultats}

Tras el estudio elaborado al respecto del tratamiento de sus audiotecas por parte de las cuatro emisoras con mayor difusión en España, podemos hablar de tres niveles de desarrollo:

-Nivel alto: RNE. Se trata de la única de las emisoras que incluye fragmentos seleccionados de su archivo sonoro desde el comienzo de su emisión («RNE Especial 75 Aniversario») en su página Web, aunque su audioteca sólo conste de archivos a partir de 2008. Además, cuenta con el sistema de búsqueda más preciso y completo, ofreciendo dos opciones de uso: una simple y otra avanzada. Una de sus mayores carencias es la ausencia de agrupación de resultados por categorías.

${ }^{2}$ Guallar, J. y Abadal, E. (2009): “Evaluación de hemerotecas de prensa digital: indicadores y ejemplos de buenas prácticas", en El Profesional de la Información mayo-junio, v. 18, n. 3, pp. 255269. 
-Nivel medio: Cadena SER. Cuenta con un sistema de búsqueda simple, aunque sí que presenta un aspecto especialmente útil para el usuario como es la agrupación de resultados por categorías, siendo el único caso de los estudiados que posee dicha posibilidad. Así mismo, su cobertura temporal es la más extensa, ofreciendo la posibilidad de acceder a archivos desde el 2003 a la actualidad.

-Nivel bajo: Onda Cero y COPE. Onda Cero cuenta con servicios prácticamente exclusivos (a excepción de RNE) como son los especiales, y su interfaz es el de mayor usabilidad para el usuario. Sin embargo, no posee un sistema de búsqueda avanzada, siendo escasas las posibilidades que nos ofrece en la mayoría de sus servicios. Además, sus archivos más longevos datan del 2010.

Sin duda es la audioteca de la COPE la que más deficiencias presenta, al no contar ni siquiera con una barra de búsqueda en su página principal. Las pestañas de acceso a su archivo sonoro no tienen la relevancia ni visibilidad adecuadas, y su sistema de presentación de resultados es el más pobre de los cuatro a analizar.

Con la llegada de Internet y la posibilidad de digitalización de sus fondos, las cadenas radiofónicas debieron decidir entre conservar su patrimonio sonoro, facilitar su difusión o ambas cosas. Al fin y al cabo, tal y como afirma Blasco Gallardo (2010: 73): «Todo Internet es un gran heredero de la archivística, por lo tanto ¿cómo podemos seguir abordando la Internet sin entender la archivística? ». Pero, ¿hasta que punto las emisoras españolas trabajan en el reto por conservar, divulgar, brindar acceso y dar valor añadido a sus archivos sonoros? ¿De qué elementos o servicios aún carecen sus audiotecas?

Tabla 2. Propuesta de Audioteca Radiofónica Digital

\section{Aspectos Generales}

\begin{tabular}{|c|c|}
\hline INDICADORES & \\
\hline Denominación & $\begin{array}{l}\text { Sería conveniente unificar términos entre las propias } \\
\text { emisoras, de tal forma que se creara un «lenguaje» propio, } \\
\text { común y fácilmente identificable por parte de los usuarios. }\end{array}$ \\
\hline $\begin{array}{l}\text { Ubicación del acceso } \\
\text { en la web a la } \\
\text { audioteca y/o } \\
\text { buscador }\end{array}$ & $\begin{array}{l}\text { Encabezando la página principal ha de estar una barra } \\
\text { buscadora y el acceso a la búsqueda avanzada. } \\
\text { De igual forma, las pestañas que nos deriven a la Audioteca y } \\
\text { los Podcast han de ocupar un lugar destacado y fácil } \\
\text { visibilidad y acceso en la página inicial del site. }\end{array}$ \\
\hline Cobertura temporal & $\begin{array}{l}\text { Resultaría imprescindible apostar por un proceso de } \\
\text { digitalización de todo el archivo sonoro que la cadena } \\
\text { conserve, así como su accesibilidad al público, siendo clave } \\
\text { este factor a la hora de considerar su audioteca desde un } \\
\text { punto de vista de SVA. }\end{array}$ \\
\hline
\end{tabular}




\begin{tabular}{|c|l|}
\hline Coste & $\begin{array}{l}\text { En la actualidad, ninguna de las emisoras nacionales saca } \\
\text { mayor partido económico de su presencia en la red que el } \\
\text { proveniente del ámbito publicitario. Sería interesante, pues, } \\
\text { tomar ejemplo de las algunas de las iniciativas que su } \\
\text { homólogo en prensa está comenzando a tomar. }\end{array}$ \\
\hline \multirow{5}{*}{ Otros servicios en la } & $\begin{array}{l}\text { Se trata de uno de los puntos clave para conseguir dotar a } \\
\text { este servicio de un valor añadido. } \\
\text { En la actualidad, RNE y Onda Cero son las únicas emisoras } \\
\text { que ofertan contenido relacionado con «Especiales» o } \\
\text { «Archivo Histórico», y Cadena SER y Onda Cero las únicas } \\
\text { que emiten una programación exclusivamente online, dos de } \\
\text { las vías que podrían convertirse en el «germen»de la } \\
\text { verdadera ciberradio de calidad por la que tal vez los usuarios } \\
\text { estarían dispuestos a pagar. }\end{array}$ \\
\hline
\end{tabular}

\section{Sistemas de consulta}

\begin{tabular}{|c|c|}
\hline INDICADORES & \\
\hline $\begin{array}{l}\text { Operadores de } \\
\text { consulta }\end{array}$ & $\begin{array}{l}\text { Sólo podremos hablar de un sistema de consulta con } \\
\text { prestaciones avanzadas si incluye funciones que permitan } \\
\text { combinar «términos mediante la utilización de operadores } \\
\text { booleanos, búsqueda de frases literales, operadores de } \\
\text { proximidad y la utilización de paréntesis para aumentar las } \\
\text { combinaciones (...) [siendo de nivel medio] si permite al } \\
\text { menos la búsqueda con booleanos y la frase literal» (Guallar } \\
\text { y Abadal, 2009:261). }\end{array}$ \\
\hline Acotación temporal & $\begin{array}{l}\text { Consideramos fundamental que el usuario pueda saber a } \\
\text { ciencia cierta qué archivos va a poder encontrar o no desde } \\
\text { el punto de vista cronológico, algo que en la actualidad es } \\
\text { imposible de averiguar de otra forma que no sea la } \\
\text { navegación dentro de cada uno de los programas. } \\
\text { Es necesario que en este punto de la búsqueda, las cadenas } \\
\text { dejen de mostrar calendarios virtuales hipervinculado de los } \\
\text { años en los que no ofertan documentación sonora. }\end{array}$ \\
\hline $\begin{array}{l}\text { Acotación en } \\
\text { colecciones, campos } \\
\text { determinados y por } \\
\text { formatos multimedia }\end{array}$ & $\begin{array}{l}\text { Si realmente queremos evidenciar la capacidad de las } \\
\text { audiotecas en pro de la optimización del tiempo de los } \\
\text { usuarios, a los que facilitan una «escucha a la carta», ha de } \\
\text { aumentar el interés por perfeccionar y refinar las búsquedas } \\
\text { y añadir valor a los resultados obtenidos para facilitar la } \\
\text { selección de aquellas noticias en las que esté interesado, } \\
\text { incluyendo para ellos el mayor número de variables de filtro } \\
\text { posibles. }\end{array}$ \\
\hline
\end{tabular}


Páginas de resultados

\begin{tabular}{|c|c|}
\hline INDICADORES & \\
\hline $\begin{array}{l}\text { Posibilidad de ordenar } \\
\text { y refinar los resultados }\end{array}$ & $\begin{array}{l}\text { Si resulta importante el perfeccionamiento en los sistemas de } \\
\text { búsqueda, no debe serlo en menor medida el proceso de } \\
\text { selección de resultados, ya que su composición puede } \\
\text { generar confusión si no están bien estructurados. }\end{array}$ \\
\hline $\begin{array}{l}\text { Identificación de los } \\
\text { términos de búsqueda } \\
\text { en los resultados }\end{array}$ & $\begin{array}{l}\text { Se trata de un aspecto especialmente útil cuando se } \\
\text { presentan muchos registros al usuario, ya que permite } \\
\text { valorar de manera sencilla la situación o posición que tienen } \\
\text { las palabras consultadas dentro del documento recuperado, } \\
\text { ya sea en el registro o en el documento final. } \\
\text { Los términos de búsqueda serán visibles tanto en los titulares } \\
\text { como en el cuerpo de los resultados. }\end{array}$ \\
\hline $\begin{array}{l}\text { Presentación de } \\
\text { documentos } \\
\text { relacionados }\end{array}$ & $\begin{array}{l}\text { Esta posibilidad debería estar contemplada desde la página } \\
\text { inicial de resultados, es decir, no tendría que ser necesario } \\
\text { entrar en cada uno de los resultados para poder acceder a } \\
\text { más material relacionado. } \\
\text { Además, las emisoras podrían hacer una mayor promoción } \\
\text { de sus archivos sonoros a través de la integración de } \\
\text { resultados de la audioteca en las noticias de actualidad, lo } \\
\text { que aportaría a los usuarios una mayor profundidad y calidad } \\
\text { informativa. }\end{array}$ \\
\hline $\begin{array}{l}\text { Gestión de los } \\
\text { resultados }\end{array}$ & $\begin{array}{l}\text { La rápida extensión de las herramientas basadas en la Web } \\
\text { Social tampoco ha pasado por alto en el sector radiofónico. } \\
\text { Aunque, si bien todas las emisoras analizadas cuentan con } \\
\text { servicios a través de los cuales los usuarios pueden } \\
\text { establecer relaciones de interactividad y reactividad como } \\
\text { webs de promoción de noticias (Digg o Menéame), redes } \\
\text { sociales (Facebook o Tuenti), servicios de microblogging } \\
\text { (Twitter) o marcadores sociales (Delicious), no está tan claro } \\
\text { el seguimiento que hacen de su empleo. }\end{array}$ \\
\hline
\end{tabular}

\section{Discussió i conclusions}

\section{Discussió i conclusions}

La valoración en términos globales muestra una escasa preocupación de las emisoras nacionales por la organización y los procesos de búsqueda de sus archivos generados. Han de ser conscientes del gran valor que reúnen para sus oyentes, y no conformarse con hacerlos públicos, sino también accesibles a través de la Web, ya que una de las grandes aportaciones de la radio digital es, precisamente «el acompañamiento de la información sonora con otros servicios y con otras modalidades de acceso" (Cebrián, 2007).

En un momento en el que el principal motor económico de los medios (la publicidad) comienza a ser insuficiente, y el acceso a la información continúa siendo clave en el desarrollo social, económico e industrial de cualquier país, las emisoras no deberían dejar pasar la 
posibilidad de convertir sus audiotecas en productos informativos, documentales y de valor añadido.

Aunque la cantidad de fondos accesibles ha crecido de forma notable, sus sistemas de búsqueda para la recuperación de informaciones presentes y retrospectivas no están a la altura de sus posibilidades, ni de las necesidades de los usuarios, no valorando por completo la máxima de que «en los portales periodísticos, al igual que en cualquier portal o sitio web, tan importante es la información contenida en ellos, como el acceso que se tenga» (López Carreño y Rodríguez Muñoz, 2003: 117). Es necesario que las cadenas radiofónicas comprendan que la documentación en sí misma es un elemento de valor añadido en cuanto que, su correcta promoción, puede derivar en beneficios tales como el patrimonio, el prestigio de la marca o el sedimento histórico (Rubio Lacoba, 2010). Por otra parte, sólo un acceso organizado, dinámico, intuitivo y de calidad a través del cual el oyente pueda no sólo realizar búsquedas, sino encontrar los resultados deseados, puede ir ligado, por ejemplo, al planteamiento de una oferta de pago. Al fin y al cabo, los servicios de valor añadido (SVA) representan un registro opcional, de pago o gratuito, a partir del cual el usuario requiere una atención personal por esa aportación económica o información suministrada (Palazón, 2001).

De la misma manera, las cadenas han de someterse a un proceso de reinvención, con el objetivo de aportar nuevos formatos y sacar el máximo partido al nuevo escenario 2.0, promocionando y fidelizando nuevas audiencias a través de una propuesta personalizada de su patrimonio sonoro.

\section{Bibliografía}

Afuera, A., (1997-1998): «El Departamento de Documentación de la Ser ante el reto digital», en Cuadernos de Documentación Multimedia, 6-7.

ARBoledAS, L., (2010): "La radio española: un negocio analógico en la era digital», en Revista de Economía Política de las Tecnologías de la Información y la Comunicación, vol XII, nำ2, mayo/agosto.

ARIZA ChICHARRo, R., (1994): "La fonoteca de RNE como archivo histórico», en La Documentació als Mitjans d' Informació. Valencia: Generalitat Valenciana. pp. 303-315.

- (2004): "El Archivo de la Palabra de Radio Nacional de España», en Revista general de información y documentación, vol 14, no 2, pp. 29-58.

ARJonA MARTín, J. B., (2009): "Los nuevos canales audiovisuales basados en web: RTVE.es», en Revista ICONO 14, no 15, pp. 98-113. 
- (2010): «De web corporativa a portal audiovisual: Rtve.es», en II Congreso Internacional AE-IC. Comunicación y desarrollo en la era digital. 3-5 de febrero de 2010, Málaga.

Berelson, B., (1952): "Content Analysis», en Handbook of Social Psychology, vol I. Nueva York: Lindzey.

BERRY, R. (2006): «Will the ipod kill the radio star? Profiling podcasting as radio. Convergence», en The International Journal of Research into New Media Technologies (12), pp. 143-162.

Caldera Serrano. J., (2004): «La documentación sonora en los Sistemas de Información Documental de los Medios Audiovisuales», en el Boletín de la Asociación Andaluza de Bibliotecarios, no 74, pp. 29-39.

Cebrián HerReROS, M., (1995): Información radiofónica: mediación técnica, tratamiento y programación. Madrid: Síntesis.

- (2007): Modelos de radio, desarrollos e innovaciones. Madrid: Fragua Comunicación.

- (2008): La radio en Internet: de la ciberradio a las redes sociales y la radio móvil. Buenos Aires: La Crujía.

- (2010): Desarrollos del periodismo en Internet. Sevilla: Comunicación Social.

Chivite Navascués, S., (2008): COPE: una cadena de radio en busca de su identidad. Madrid: Fragua Comunicación.

CHOMÓn SERNA, J.M., (2011): Evolución y transformaciones de Radio 5 Todo Noticias. Una radio menos cercana. Madrid: Fragua Comunicación.

EGM, Resumen, marzo 2012. Consultado el 15 de abril de 2012 en: http://www.aimc.es/Entrega-de-resultados-EGM-1\%C2\%AA-

ola,1067.html

DíEZ, P., (2001): «El Departamento de Documentación de los Servicios Informativos de la Cadena SER en Madrid: un poco de historia» en Scire, vol 77, no 2, pp. 151-156.

FRANQUET, R., (1999): Radio y televisión en la red: el primer asalto. Barcelona: Societat Catalana de Comunicació.

GANZABAL LEARRETA, M., (2009): «De la tediosa digitalización a la revolución de la radio en la red», en Académica Question, vol 1, no 21.

Gómez, G. R., (1999): "La SER evoca sonidos con historia», en El País, 27 de septiembre de 1999. Consultado el 28 de marzo de 2012 en: http://www.elpais.com/articulo/Pantallas/ESPANA/CADENA SER/SER/ evoca/sonidos/historia/elpepirtv/19990927elpepirtv 18/Tes 
Guallar, J. y E. AbAdAL, (2008): "Les hemeroteques digitals de la premsa catalana: anàlisi dels diaris catalans de més difusió", en Jornades Catalanes de Documentació (11es: Barcelona, 22-23 maig 2008). Barcelona: COBDC, p. 149-162. Consultado el 28 de marzo de 2012 en: http://eprints.rclis.org/archive/00013686/01/jornades2008 guallaraba dal hemeroteques diaris.pdf

- (2009): «Evaluación de hemerotecas de prensa digital: indicadores y ejemplos de buenas prácticas», en El Profesional de la Información mayojunio, v. 18, n. 3, pp. 255-269.

HAYE, R., (2000): La Radio del Siglo XXI: nuevas estéticas. Buenos Aires: Ciccus, La Crujía.

Hendy, D., (2007): Radio in the global age. Cambridge: Polity Press.

López Carreño, R., (2004): "Grado de desarrollo de los portales periodísticos españoles de radio y televisión", en Scire: Representación y organización del conocimiento, vol. 10, no 2, pp. 115-122.

MaRTín MuÑOz, J. y J. LóPEz PAVILLARD, (1997-1998): «RTVE: Reorganización de la Documentación en un entorno multimedia», en Cuadernos de documentación multimedia, no 6-7.

Nuño Moral, M. V. y M. F.SánChez HeRnándeZ, (1999): «Los servicios de documentación sonora ante el reto digital», en Cuadernos de Documentación Multimedia, 8. Consultado el 5 de mayo de 2012 en: http://www.ucm.es/info/multidoc/multidoc/revista/num8/marivi.html

NUÑo MoRAL, M. V. (2007): Documentación en el medio radiofónico: hacia un entorno digital. Madrid: Síntesis.

PAUL, N., (1997): "Media libraries and new media», en Poynter Institute. Consultado el 28 de marzo de 2012 en: http://www.poynter.org/content/content view.asp?id=5693\&sid=26

Prieto, L., (2007a): «Fondos sonoros y escritos en Radio Nacional de España: un modelo de respuesta a las demandas de estructuración de la información en entornos multimedia», en Boletín de la ANABAD, Tomo 57, № 2, pp. 137-148.

- (2007b): «Información y documentación radiofónica: espacios para un interés común. La experiencia de Radio Nacional de España», en El profesional de la información, 2007, septiembre-octubre, v. 16, n. 5, pp. 443-449.

- (2009): «El Archivo Sonoro de Radio Nacional de España: interés histórico y valor documental», en Boletín de la Asociación Española de Documentación Musical, año 13, pp. 28-35. 